\title{
Broad consent for health care-embedded biobanking: understanding and reasons to donate in a large patient sample
}

\author{
Gesine Richter, MA, MBA ${ }^{1,3}$, Michael Krawczak, MSc, PhD², Wolfgang Lieb, MD, PhD ${ }^{1}$, \\ Lena Wolff, cand. med. ${ }^{3}$, Stefan Schreiber, MD, PhD ${ }^{4}$ and Alena Buyx, MD, PhD ${ }^{3}$
}

Purpose: To facilitate ethically acceptable and practically successful health care-embedded biobanking, the attitudes and understanding of patients and their motivation to participate need to be explored.

Methods: A questionnaire study was conducted among 760 outpatients of a northern German university hospital to assess their awareness of, and motivation for giving broad consent to health care-embedded biobanking, also addressing the issue of feedback on individual-level research findings.

Results: The overall willingness to give broad consent was high $(86.9 \%)$ in our study, even though the subjective and objective understanding of patients was found to be only modest. Most participants who consented did so for prosocial reasons (altruism, solidarity, reciprocity, gratitude), whereas self-interest or worries about disadvantages played only a marginal role. Better objective understanding was associated with both a greater demand for feedback on individual research findings and a higher willingness to consent. Intermittent modification of the information material provided by the hospital led to significantly improved objective understanding.

Conclusion: Patient willingness to give broad consent to health care-embedded biobanking is high, with prosocial reasons driving decision making more than factual knowledge and approval or disapproval of specific consent elements. Future efforts to improve the information material used in health care-embedded biobanking should therefore emphasize prosocial reasons to consent.

Genet Med advance online publication 22 June 2017

Key Words: broad consent; health care-embedded biobanking; understanding

\section{INTRODUCTION}

Biorepositories for medical research aim at the long-term storage of human biomaterial alongside associated data. Such collections are usually established for specific research projects. More recently, however, the large-scale collection and storage of samples left over from clinical routine has become popular in an approach termed health careembedded (or health care-integrated) biobanking. ${ }^{1,2}$ Although residual biomaterial from the clinic has been preserved for research purposes in the past, ${ }^{3}$ its systematic collection in large patient cohorts is a novel development. University hospitals, in particular, increasingly engage in health care-embedded biobanking. ${ }^{4}$

Health care-embedded, hospital-based biobanking differs from other forms of biobanking in that donors are not recruited among healthy volunteers or patients outside the clinic, but get involved because they seek diagnosis or treatment. ${ }^{5}$ Moreover, the intended use of residual biomaterial is not normally confined to a specific research project or period of time. These differences have important impact, not only in terms of the legal and ethical framework of biobanking but also on the perception of patients and on their willingness to consent (for more information, see refs. 1,2,6).

Since most scientific questions that can potentially be addressed by health care-embedded biobanking are unknown at the time of collection, the paradigm of informed consent from clinical research cannot be transferred easily to this type of research. This limitation has already been recognized..$^{7-10}$ Recently, broad consent was thus recommended, at both national ${ }^{11}$ and international levels, ${ }^{12}$ as an ethical option. Broad consent entails the provision of general information about the particular biobanking activity, but does not specify any individual research projects. This way, it provides researchers with sufficient flexibility to pursue a wide range of future, scientific agendas. ${ }^{13}$ In most-but not all ${ }^{14,15}$ instances, broad consent also implies that the patient acknowledges that they will not receive any feedback on incidental findings.

Although broad consent seems to have become the new standard for biobanking, both its ethical justification and its acceptability are still contested. As regards ethical justification, it is thus discussed whether narrower consent forms

${ }^{1}$ Institute of Epidemiology, Christan-Albrechts-Universität zu Kiel, Kiel, Germany; ${ }^{2}$ Institute of Medical Informatics und Statistics, Christan-Albrechts-Universität zu Kiel, Kiel, Germany; ${ }^{3}$ Division of Biomedical Ethics, Institute of Experimental Medicine, Christian-Albrechts-Universität zu Kiel, Kiel, Germany; ${ }^{4}$ Institute of Clinical Molecular Biology, Christian-Albrechts-Universität zu Kiel, Kiel, Germany. Correspondence: Alena Buyx (medizinethik@iem.uni-kiel.de) 
would be ethically preferable to broader forms, or vice versa, and which findings, if any, should be reported to patients. $^{7-10,16}$ Regarding ethical acceptability, it is still unclear whether patients are willing to give broad consent in the first place, whether they truly understand the concept, and why they ultimately either give or withhold broad consent. ${ }^{17}$

In view of the rapidly increasing popularity of health careembedded biobanking, a detailed empirical study to inform the discussions about the ethical justification and acceptability of broad consent seems well warranted. In other forms of biobanking, the ability of donors to fully understand the meaning and consequences of broad consent has been questioned, ${ }^{18-20}$ and several studies revealed that the understanding of different consent models is often poor. ${ }^{21,22}$ Moreover, previous research into the motivation of participants to give broad consent $t^{3-5,23-26}$ either was not focused on health care-embedded biobanking, or assessed hypothetical scenarios, or investigated small samples. To ensure that health care-embedded biobanking with broad consent is both ethically acceptable and practically successful in large patient cohorts, the proportion of patients giving or withholding broad consent and the reasons for their decision need to be explored, including their relation to the level of comprehension of broad consent, in the immediate care context. Whether the non-reporting of findings is acceptable to patients also needs to be assessed in real-world settings.

Since such research is still lacking, we examined decisionrelevant aspects of comprehension and motivation in a large cohort of patients involved in a pilot implementation of broad consent-based health care-embedded biobanking in northern Germany $(n=760)$. To the best of our knowledge, our study is the first to appraise these aspects in a large sample and "in the field," i.e., at the time of consenting within a regular healthcare setting, thereby filling an important gap in the literature.

\section{MATERIALS AND METHODS}

Following approval by the local institutional review board, a total of 760 adult patients were approached at the Comprehensive Center for Inflammation Medicine (CCIM) in Kiel, Germany, in September 2015 and in February 2016, and asked to participate in the present study. The CCIM is an outpatient clinic for inflammatory conditions at the University Hospital Schleswig-Holstein (UKSH), the largest health-care provider in northern Germany (150,000 patients per year). UKSH Campus Kiel is currently implementing health careembedded biobanking following a newly developed broad consent policy. The CCIM patient population can be assumed to fully reflect the sociodemographic distribution of all outpatients at UKSH Campus Kiel and was therefore chosen to pilot the rollout of the broad consent. Patients were provided with both an informational brochure and the consent form at the time of admission. Study participants were additionally asked to fill out a questionnaire designed to evaluate their understanding of the written material and to ascertain their motivation to consent or not.
The information brochure and consent form were developed in accordance with recommendations by TMF, the German umbrella organization for networked medical research. ${ }^{27,28}$ Both documents are in accordance with templates issued by the Standing Working Group of the German Research Ethics Committees ${ }^{11}$ but tailored for use by UKSH Campus Kiel.

In addition to explaining the general purpose of health care-embedded biobanking, the information brochure specified that no additional biomaterial would be taken and that consent would cover only the use of leftover biomaterial. Patients were also informed that they would not benefit personally from participating and that they would not suffer any disadvantages if they declined participation. Special emphasis was put on the fact that leftover biomaterial and associated clinical data would be stored indefinitely and used for unspecified research purposes unlimited in terms of time, scope, and methodology (broad consent). Participants were also informed that they would not receive feedback on individual research-derived findings, irrespective of their potential clinical utility (nonreporting policy). Patients were given the choice to either give broad consent with no feedback, or to withhold consent entirely. The brochure also highlighted that all research covered by the broad consent would be reviewed by the local institutional review board. The consent form used standard language to recapitulate the main issues of the information brochure and required the patient's signature. Patients had the opportunity to discuss the documents with trained staff at the CCIM.

Randomly selected patients were then asked to answer a standardized questionnaire to assess their individual levels of understanding of the consent materials. The study was carried out in two phases: After a first evaluation round in September $2015(\mathrm{E} 1 ; n=425)$, the brochure was rewritten to improve the language. It included sections about nonreporting, benefit to patients, access to biomaterial and data by non-UKSH researchers, and issues of privacy and data protection. The text was not changed in terms of content but only improved in clarity (e.g., by altering the order or length of sentences, simplifying punctuation and grammar, and cutting superfluous words). A second set of patients who received the improved brochure was subsequently enrolled in the study in February 2016 (E2; $n=335)$.

\section{Questionnaire}

A questionnaire was developed and subjected to several quantitative pretests to determine its appropriate length and comprehensibility. It comprised 23 items, including 11 Likert scale items, 3 statement items, 2 open items, and 6 sociodemographic questions. The questionnaire was divided into four sections on understanding, motivation, incidental findings, and sociodemographic characteristics (see Supplementary Material online).

The section on patient understanding comprised both a subjective self-assessment of understanding and potential reasons for lack of understanding. In addition, it contained 
Likert scales and statement items to test the objective level of understanding. The eight Likert scale questions used a threepoint scale ("applies," "does not apply," "I do not know").

The section on motivation provided space to input reasons for not consenting and a statement item with 12 statements about the possible motivation for consenting (several responses allowed).

The fourth section ascertained the attitude of patients toward nonreporting of findings, an issue that was pondered intensely by the proponents of health care-embedded biobanking in Kiel. Participants were asked about their personal preference regarding the reporting of findings, and whether they deemed appropriate the current practice in Kiel of not reporting any findings, even if these were potentially health-relevant.

\section{Statistics}

Differences in proportions were assessed for statistical significance $(P<0.05)$ by way of $\chi^{2}$ tests. The relationship between potential predictors and dichotomous response variables was analyzed by means of logistic regression modeling with backward variable selection employing the Wald $t$-test. The distributions of quantitative variables were compared between subgroups using a Wilcoxon test. All statistical analyses were carried out with SAS STAT version 9.4 (SAS Institute, Cary, NC).

\section{Consent and understanding}

\section{RESULTS}

The project, undertaken as a delivery-and-collection questionnaire study, achieved a very high level of participation. Of

\begin{tabular}{lcccc}
\multirow{2}{*}{$\begin{array}{c}\text { Table 1 Demographic characteristics of study participants } \\
\text { Age (years) }\end{array}$} & $\begin{array}{c}\text { Phase E1 } \\
\text { Phase E2 }\end{array}$ \\
\cline { 2 - 5 } & Male (\%) & \begin{tabular}{c} 
Female (\%) \\
\cline { 5 - 5 }
\end{tabular} & Male (\%) & Female (\%) \\
\hline $18-30$ & $21(19.8)$ & $38(20.0)$ & $19(13.9)$ & $14(12.0)$ \\
\hline $31-45$ & $20(18.9)$ & $28(14.7)$ & $15(11.0)$ & $17(14.5)$ \\
$46-60$ & $34(32.1)$ & $68(35.8)$ & $30(21.9)$ & $26(22.2)$ \\
$61-75$ & $27(25.5)$ & $45(23.7)$ & $60(43.8)$ & $44(37.6)$ \\
$76-90$ & $4(3.8)$ & $11(5.8)$ & $13(9.4)$ & $16(13.7)$ \\
\hline Total (\%) & $106(35.8)$ & $190(64.2)$ & $137(53.9)$ & $117(46.1)$ \\
\hline
\end{tabular}

aphase- and sex-specific percentage of patients in the respective age group.

${ }^{b}$ Phase-specific percentage of patients of the respective sex.
760 delivered questionnaires, 749 (98.6\%) were returned, and 550 of these $(73.4 \%)$ were completed. The response rate increased from 69.6\% (296 completed of 425 delivered) in phase E1 to $75.8 \%$ in E2 (254 of 335). In both phases, age distribution was similar in the two sexes (Table 1). However, significantly more men were included in phase E2 (53.9\%) than in phase E1 $\left(35.8 \% ; \chi^{2}=18.212\right.$, 1.d.f., $\left.P<0.0001\right)$. Age distribution was also found to differ significantly between phases $\left(\chi^{2}=31.181\right.$, 4.d.f., $\left.P<0.0001\right)$ mainly because a larger proportion of older-aged patients ( $>60$ years) was included in phase E2 (52.4\%) than in phase E1 (29.4\%). Regarding level of education, the two samples were found to be well representative of the general German population (Table 1).

The willingness to provide broad consent for health careembedded biobanking was high in our study $(n=478 ; 86.9 \%)$. In phase E1, 244 of 296 patients (82.4\%) consented even though only 184 of them (62.2\%) stated that they found the information material sufficiently intelligible (subjective understanding). When testing objective understanding in phase E1 (multiple responses possible, Table 2), the following issues were found to have been poorly understood: nonreporting of incidental findings (37.5\%), scientific scope of data and biomaterial use (34.8\%), possible use by external researchers (35.1\%), and absoluteness of data protection (21.0\%). Much better levels of objective understanding were achieved regarding the right to withdraw $(71.6 \%)$ and the lack of immediate personal benefit from consenting (52.4\%). No notable differences in objective understanding were observed between the group of patients who stated that they found the material sufficiently intelligible and the overall sample (data not shown).

In view of the poor level of understanding in phase E1, the information brochure was revised to improve the clarity of its language before a second group of patients was enrolled. In phase E2, an even higher proportion of participants who returned the form gave broad consent $(n=234,92.1 \%)$ even though, again, only two-thirds of those who consented (155; $66.2 \%)$ stated that they found the information material sufficiently intelligible (subjective understanding). This notwithstanding, our intermittent text revision appears to have had a considerable impact (Table 2). The level of objective understanding in phase E2 was either significantly higher

Table 2 Understanding of selected topics of the broad consent documents

\begin{tabular}{|c|c|c|c|}
\hline Topic & Phase E1 $(n=296)$ & Phase E2 $(n=254)$ & $P$ value \\
\hline Reporting of incidental findings & $111(37.5 \%)$ & $165(65.0 \%)$ & $<0.0001$ \\
\hline Scientific scope of use ${ }^{a}$ & $103(34.8 \%)$ & $117(46.1 \%)$ & 0.0072 \\
\hline Right to withdraw ${ }^{a}$ & $212(71.6 \%)$ & $164(64.6 \%)$ & 0.076 \\
\hline Use by external researchers ${ }^{a}$ & $104(35.1 \%)$ & $81(31.9 \%)$ & 0.422 \\
\hline Absoluteness of data protection & $62(21.0 \%)$ & $142(55.9 \%)$ & $<0.0001$ \\
\hline Personal benefit & $155(52.4 \%)$ & $170(66.9 \%)$ & 0.0005 \\
\hline
\end{tabular}

${ }^{\text {aTh }}$ This topic was addressed in the questionnaire by the provision of two contradictory statements; incorrect affirmation of at least one statement or nonaffirmation of both statements was counted as a wrong answer.

The $p$ value corresponds to a $\chi^{2}$ test for different proportions with one degree of freedom. 
(incidental findings, scientific scope, data protection, personal benefit) or only insignificantly worse (right to withdraw, external use) than in phase E1. Overall, the mean number of correct answers increased from 2.52 in phase E1 to 3.30 in phase E2 (Wilcoxon $\mathrm{z}=5.440, P<0.0001$ ). It must be noted, however, that this improvement was accompanied by a significant increase in the mean number of unanswered questions (E1: 2.47, E2: 2.86; Wilcoxon $\mathrm{z}=4.836, P<0.0001$ ).

As noted above, the two phases of our study differed significantly in terms of the underlying sex and age distributions, so that the observed improvement in understanding may partly reflect confounding. Therefore, we performed linear regression analysis treating the study phase as the independent, and the number of correct answers as the dependent, variable, adjusting for patient sex, age, and education level. In addition to study phase $(t=3.956, P<0.0001)$, only education $(t=6.828, P<0.0001)$ was found to be of significant influence on the level of objective understanding (i.e., number of correct answers given), but not sex (Wald $t=0.441, P=0.660)$ or age $(t=-0.645, P=0.519)$. An increase in education level by one ordinal unit increased the mean number of correct answers by 0.376 (standard error: 0.055).

Finally, to identify factors that might have influenced decision making, we undertook logistic regression analysis of the following independent variables: sex, age, education, number of correct answers, number of unanswered questions, study phase, and two statements about whether the patient graded the information material as (i) sufficiently intelligible and (ii) of appropriate length. Backward variable selection retained only the number of unanswered question as a statistically significant predictor $\left(\chi^{2}=4.983\right.$, 1.d.f., $\left.P=0.026\right)$, with an estimated odds ratio (OR) for consenting of $0.86(95 \%$ CI: 0.75-0.98) per unanswered question.

\section{Feedback on research results}

The information material stated explicitly that no research results would be returned to patients, irrespective of their clinical relevance. This notwithstanding, the questionnaire asked which kind of feedback participants would have wished to receive if they had had a choice, and whether participants thought that not giving any feedback was appropriate.

Participants could choose between four potential forms of feedback: no feedback, feedback only on results of indisputable clinical relevance, feedback only on results of at least probable clinical relevance, or feedback on any results (Table 3). In phase E1, the attitude of patients was diverse in that almost equal proportions of participants preferred feedback at the three different levels of clinical validity, with a minority preferring no results. In stark contrast to this, nearly three-quarters of patients in phase E2 would have wished to be informed about any results. No notable difference in terms of their attitude toward feedback was observed between patients who did and those who did not consent (data not shown).

In agreement with the personal preferences expressed, a much higher proportion of patients in phase E2 (71.3\%) than E1 (48.0\%) opposed the stringent nonreporting policy pursued at UKSH Campus Kiel (Table 3). In fact, logistic regression analysis treating opposition or not as the dependent variable, and study phase and personal preference as independent variables, revealed a highly significant effect of the latter $\left(\chi^{2}=15.836\right.$, 1.d.f., $\left.P<0.0001\right)$ but not the former predictor $\left(\chi^{2}=2.042\right.$, 1.d.f., $\left.P=0.153\right)$. The OR for opposition was 1.54 per unit decrease in preferred validity level (95\% CI: $1.25-1.91$ ), i.e., a patient was $50 \%$ more likely to oppose the nonreporting policy if their wish for feedback was attached to the next lower level of clinical validity of the result.

In summary, the improved level of understanding in phase E2 appears to have resulted in a stronger inclination of participants to disagree with the nonreporting policy. Interestingly, however, opposition had no adverse effect on decision making, because the proportion of opposing patients was even slightly higher among those who did consent (296 of $478 ; 61.9 \%)$ than among those who did not (26 of $48 ; 54.2 \%)$.

Table 3 Attitude toward feedback on research findings among consenting participants

Question/Answers

Phase E1 $(n=296)$

Phase E2 $(n=254)$

\begin{tabular}{|c|c|c|}
\hline \multicolumn{3}{|c|}{$\begin{array}{l}\text { Research using biomaterial may lead to results that could be relevant to your personal health. However, most of these results are either of low quality or } \\
\text { may turn out to be incorrect. If you had a choice, what kind of feedback would you wish to receive? }\end{array}$} \\
\hline None at all & $14(4.7 \%)$ & $15(5.9 \%)$ \\
\hline Only on results of obvious clinical relevance & $66(22.3 \%)$ & $17(6.7 \%)$ \\
\hline On any results & $83(28.0 \%)$ & $187(73.6 \%)$ \\
\hline No answer given & $55(18.6 \%)$ & $9(3.5 \%)$ \\
\hline Yes & $63(21.3 \%)$ & $21(8.3 \%)$ \\
\hline Not sure & $56(18.9 \%)$ & $51(20.1 \%)$ \\
\hline No & $142(48.0 \%)$ & $181(71.3 \%)$ \\
\hline No answer given & $35(11.8 \%)$ & $1(0.4 \%)$ \\
\hline
\end{tabular}




\section{Motivation to give broad consent}

When asked about their motivation (motivational items M01M12), we found that most participants who consented did so mainly for prosocial reasons, including altruism (M01-M03), solidarity (M04, M05), reciprocity (M06), and gratitude (M07) (terms defined according to ref. 29). Self-interest (M08) or the drive for setting an example (M09) did not play a dominant role, and only very few participants worried about disadvantages if they did not consent (M10), referred to the experience of others (M11), or had no specific reason for consenting at all (M12) (Table 4).

Several significant differences in terms of patient motivation became apparent between the two phases (Table 4). In order to determine which individual-specific factors may potentially explain these, we performed logistic regression analyses treating each motivation item separately as the dependent variable and female sex, age, education level, past experience with medical research, objective understanding (i.e., number of correct answers), expectation of personal benefit, and opposition to the nonreporting policy as independent variables. The following statistically significant effects were observed upon multivariate analysis with backward selection: M01, female sex (OR: 1.78, 95\% CI: 1.23-2.59), age (OR: 0.79, 95\% CI: 0.68-0.93); M02, understanding (OR: 1.37, 95\% CI: 1.21-1.56); M03, understanding (OR: 1.17, 95\% CI: $1.02-$ 1.36); M06, understanding (OR: 1.21, 95\% CI: 1.08-1.37); M07, age (OR: $1.29,95 \%$ CI: 1.10-1.51), expectation of personal benefit (OR: 0.55, 95\% CI: 0.34-0.91); M08, expectation of personal benefit (OR: 2.39, 95\% CI: 1.40-4.06), age (OR: 0.75, 95\% CI: 0.62-0.91); M09, understanding (OR: 1.23, 95\% CI: 1.07-1.43; for a complete list of univariate ORs and details on the coding of nonbinary independent variables, see Supplementary Table S1). With one exception, however, inclusion of study phase as an independent variable did not point toward strong confounding of the observed phase differences in motivation (Table 4) by the abovementioned predictors. Only for motivation item M06 did joint consideration of the level of understanding and study phase render the influence of the latter on motivation statistically insignificant $\left(\chi^{2}=2.207\right.$, 1.d.f., $\left.P=0.137\right)$.

Taken together, self-interested reasons to donate played a significant role only for those participants who had mistaken the donation of biomaterials to imply personal benefit. All other participants had mainly prosocial reasons, with women being more inclined to state the support of research as a motivation (altruism); and the elderly being more inclined to donate out of gratitude to their doctors. Those with better objective understanding were even more likely to state reciprocity or altruism as a reason for consenting.

\section{DISCUSSION}

Recent papers have emphasized the need to study broad consent in health care-embedded biobanking and reasons affecting donors' willingness to consent. ${ }^{8,30}$ To the best of our knowledge, our study is the first to assess understanding and attitudes regarding broad consent in health care-embedded biobanking in a large sample of patients seeking treatment in a university hospital.

Overall, there was positive acceptance of broad consent in our sample (up to 92\%). This level of agreement is in line with levels for previous studies on broad consent in other forms of biobanking (e.g., ref. 31), and it is encouraging to see that the willingness of patients to support research extends to health care-embedded biobanking as well. The first conclusion from our study is, therefore, that patients generally accept a broad consent policy when confronted with it and agree with the unlimited storage and broad use of their samples and data for medical research.

Several studies on other forms of biobanking revealed that the general understanding of consent forms is poor. ${ }^{20,22,32-34}$ Not surprisingly, our findings indicate that both subjective and objective understanding is modest in health careembedded biobanking as well. However, we also noted a substantial improvement of the understanding of specific consent components upon textual modification. Therefore, our data highlight that clarity of language can have a

Table 4 Motivation to consent of participants who actually consented

\begin{tabular}{|c|c|c|c|c|c|}
\hline & Type & Motivation item & Phase E1 $(n=244)$ & Phase E2 $(n=234)$ & $P$ value \\
\hline M01 & Altruism & Support of research in general & $188(77.1 \%)$ & $48(20.5 \%)$ & $<0.0001$ \\
\hline M02 & & Helping all future patients & $120(49.2 \%)$ & $131(56.0 \%)$ & 0.137 \\
\hline M03 & & Interest in research and want to be part of it & $63(25.8 \%)$ & $45(19.2 \%)$ & 0.085 \\
\hline M04 & Solidarity & Helping future patients with same disease & $157(64.3 \%)$ & $89(38.0 \%)$ & $<0.0001$ \\
\hline M05 & & Feeling connected with future patients & $55(22.5 \%)$ & $32(13.7 \%)$ & 0.012 \\
\hline M06 & Reciprocity & Returning own benefit from research & $118(48.4 \%)$ & $92(39.3 \%)$ & 0.046 \\
\hline M09 & & Acting as a role model & $64(26.2 \%)$ & $49(20.9 \%)$ & 0.174 \\
\hline M10 & & Worry about disadvantages if not consenting & $9(3.7 \%)$ & $10(4.3 \%)$ & 0.744 \\
\hline M11 & & Knowing of others who consented & $8(3.3 \%)$ & $22(9.4 \%)$ & 0.058 \\
\hline M12 & & No specific reasons & $5(2.1 \%)$ & $11(4.7 \%)$ & 0.107 \\
\hline
\end{tabular}

The $P$ value corresponds to a $\chi^{2}$ test for different proportions ( 1 d.f.). 
significant effect on understanding. This is in contrast to previous work in biobanking, where an improvement in language did not lead to better understanding. ${ }^{35}$ One possible explanation for this apparent discrepancy is that, owing to the lack of memory bias in our study, we were able to test language-dependent understanding more specifically. Our data therefore show that, with careful attention paid to syntax and semantics in information material, understanding of the characteristics of health care-embedded biobanking can be improved.

Our data also suggest that better understanding increases the willingness to give broad consent, even with nonreporting of findings. The consent rate was higher in phase E2, where objective understanding was better. The provision of concise and intelligible information documents should therefore be in the self-interest of those planning health care-embedded biobanking. Interestingly, however, the level of self-perceived understanding in phase E2 did not improve to the same degree as did the level of objective understanding. This finding corroborates the current perception of the understanding of information material by research participants. ${ }^{32}$ Moreover, more questions were left unanswered in phase E2 than in phase E1. Since E2 exhibited a higher consent rate than E1, such a sign of uncertainty seems to contradict the fact that the number of unanswered questions was the only statistically significant predictor of the consent decision. In fact, the likelihood of consenting decreased by roughly $20 \%$ with every test item left unresolved. One possible explanation for this incongruity is that the effects of different answering behaviors in phases E1 and E2 were overcompensated by the large number of phase differences pertaining to patient motivation itself. In any case, our findings underline that, in addition to its ensuring better objective understanding by patients, their willingness to get involved would also benefit from an improved subjective understanding of health careembedded biobanking, for example, by targeted educational campaigns.

The policy on nonreporting individual-level research results to patients had been an issue of vigorous debate when the broad consent process was being devised in Kiel. In our study, we observed that better objective understanding was significantly associated with a negative attitude toward nonreporting. The better patients understood the specifics of health care-embedded biobanking, the more poignantly did they express their wish to receive feedback on results. However, a negative attitude toward nonreporting did not prevent patients from giving consent at a higher rate than those with less pronounced views on nonreporting. In other words, better objective understanding may lead to a clearer attitude of patients toward existing policies, but this does not negatively affect their willingness to consent. Being associated with better rather than worse objective understanding, this kind of acquiescence cannot be dismissed as an artifact of poor comprehension. Instead, it appears likely that the prosocial motives of patients to consent to health careembedded biobanking may have overridden any educated objection to the withholding of information relevant to patients' health.

Indeed, most participants who consented did so mainly for prosocial reasons, including altruism, solidarity, reciprocity, and gratitude. Self-interest did not play a dominant role, and only very few participants worried about disadvantages in case they did not consent, or had no specific reason for consenting at all. These attitudes were very robust across study phases and only a few influencing factors were discernible, including female sex as a factor predisposing for altruistic reasons, and gratitude toward doctors being more important with increasing age. Objective understanding was found to influence motivation insofar as patients with better understanding were more likely to cite reciprocity or altruism as reasons to donate (despite their more pronounced objection to nonreporting).

\section{Limitations}

Therapeutic misconception is known to be a complication of biomedical research studies; ${ }^{36}$ however, we regard this as an unlikely impediment to the present study. The expectation of personal benefit played such a minor role for patients that it is likely a majority were aware that donating would not have influenced the quality of personal diagnosis and treatment. Second, in our study, patients did not have a choice between broad consent and narrower forms of consent. As such, we can draw conclusions regarding overall acceptability of an established broad consent policy for health care-embedded biobanking, and how understanding and attitudes relate to patients' willingness to give such consent, but not if patients would have preferred narrower consent forms if they had had a choice. Third, even though our sample was representative of the outpatient population in Kiel, where a high response rate was achieved, our results are not directly transferable to other clinical or cultural backgrounds. That said, even though the absolute response figures achieved may differ from one setting to the next, we believe that the qualitative results of our study will hold true for health care-embedded biobanking in general. Finally, some uncertainty remains as to the extent to which the improvement in objective understanding between the two phases of our study was due to the revision of the information material. Only long-term experience will allow the identification of other, unmeasured cofactors that may have played a role in this regard.

\section{Conclusion}

Our study showed the overall ethical acceptability of broad consent in health care-embedded biobanking. With careful attention paid to the wording of concise information material, it is possible to achieve good objective understanding of health care-embedded biobanking. Overall, there is great willingness of patients to give broad consent to the collection of leftover biomaterial and the use of routine data for research. Moreover, the better the understanding of patients, the higher is their willingness to get involved. Prosocial reasons appear to play a major role, whereas self-interest and worries about disadvantages seem to be of minor importance 
for consenting. Altruism, reciprocity, solidarity, and gratitude were found to be more relevant for decision making than objective or subjective knowledge, or objection to particular elements of the broad consent process, including nonreporting of findings. Therefore, future efforts to improve the information material used in health care-embedded biobanking should emphasize prosocial motivation, instead of focusing on the amount and precision of information conveyed and the assuagement of fears of disadvantages.

\section{SUPPLEMENTARY MATERIAL}

Supplementary material is linked to the online version of the paper at http://www.nature.com/gim

\section{ACKNOWLEDGMENTS}

The authors gratefully acknowledge the kind support of the pilot phase by all academic and nonacademic staff of the Comprehensive Centre for Inflammation Medicine (CCIM), University Hospital Schleswig-Holstein Campus Kiel, and thank all patients for their participation. This work was supported by the German Ministry of Education and Research (grant 01EY1103 to the PopGen 2.0 Network, P2N).

\section{DISCLOSURE}

The authors declare no conflict of interest.

\section{REFERENCES}

1. Kaye J. Embedding biobanks in a changing context. In: Kaye J, Gibbons SMC, Heeney C, Parker M, Smart A, (eds). Governing Biobanks: Understanding the Interplay between Law and Practice. Hart Publishing: Oxford, UK, 2012:30-51.

2. Kaye J. Embedded biobanks as tools for personalised medicine. Norsk Epidemiologi 2012;21:169-175.

3. Chan TW, Mackey S, Hegney DG. Patients' experiences on donation of their residual biological samples and the impact of these experiences on the type of consent given for the future research use of the tissue: a systematic review. Int J Evid Based Healthc 2012;10:9-26.

4. Mitchell D, Geissler J, Parry-Jones A, et al. Biobanking from the patient perspective. Res Involve Engage 2015;1:4.

5. Vermeulen E, Schmidt M, Aaronson N, et al. Opt-out plus, the patients' choice: preference of cancer patients concerning information and consent regimen for future research with biological samples archived in the context of treatment. J Clin Pathol 2009; 62:275-278.

6. Riegman $\mathrm{PH}$, van Veen EB. Biobanking residual tissues. Hum Genet 2011;130:357-368.

7. Cargill SS. Biobanking and the abandonment of informed consent: an ethical imperative. Public Health Ethics 2016;9:1-9.

8. Grady C, Eckstein L, Berkman B, et al. Broad consent for research with biological samples: workshop conclusions. Am J Bioeth 2015;15:34-42.

9. Hofmann B, Solbakk JH, Holm S. Consent to biobank research: one size fits all? In: Solbakk JH, Holm S, Hofmann B (eds). The Ethics of Research Biobanking. Springer: Heidelberg, Germany, 2009, pp 3-23.

10. Koenig BA. Have we asked too much of consent? Hastings Cent Rep 2014;44:33-34.

11. Permanent Working Party of the German Medical Ethics Committees. Template for informed consent concerning the donation, storage, and utilization of biological materials as well as collecting, processing, and usage of (related) data in biobanks (approved by the General Assembly on 09.11.2013). Available at http://www.ak-med-ethik-komm.de/index. php?option = com_content\&view $=$ article\&id $=145 \&$ ltemid $=154 \&$ lang $=$ de. Accessed on October 2, 2016.

12. World Medical Association (WMA). Request for Comments on WMA Declaration on Ethical Considerations regarding Health Databases and Biobanks (Draft). Available at http://www.wma.net/en/ 20activities/10ethics/15hdpublicconsult. Accessed on October 2, 2016.
13. Strech D, Bein S, Brumhard $M$ et al. A template for broad consent in biobank research. Results and explanation of an evidence and consensusbased development process. Eur J Med Genet 2016;59:295-309.

14. National Institutes of Health. The All of Us Research Program. Available at https://www.nih.gov/research-training/allofus-research-program. Accessed on 27 February 2017

15. Darnell AJ, Austin H, Bluemke DA et al. A clinical service to support the return of secondary genomic findings in human research. Am J Hum Genet 2016;98:435-441.

16. Richter G, Buyx A. Broad consent for biobanking research-the ethical debate, German. Eth Med 2016;28:311-325.

17. Garrison NA, Sathe NA, Antommaria AHM et al. A systematic literature review of individuals' perspectives on broad consent and data sharing in the United States. Genet Med 2016;18:663-671.

18. Beskow LM, Dombeck CB, Thompson CP, Watson-Ormond JK, Weinfurt KP. Informed consent for biobanking: consensus-based guidelines for adequate comprehension. Genet Med 2015;17:226-233.

19. Wendler D, Grady C. What should research participants understand to understand they are participants in research? Bioethics 2008;22: 203-208.

20. Appelbaum PS. Understanding "understanding": an important step toward improving informed consent to research. AJOB Prim Res 2010;1:1-3.

21. D'Abramo F, Schildmann J, Vollmann J. Research participants' perceptions and views on consent for biobank research: a review of empirical data and ethical analysis. BMC Medical Ethics 2015;16:60-70.

22. Sand K, Kaasa S, Loge JH. The understanding of informed consent information-definitions and measurements in empirical studies. $A J O B$ Prim Res 2010;1:4-24.

23. Sanderson SC, Brothers KB, Mercaldo ND et al. Public attitudes toward consent and data sharing in biobank research: a large multi-site experimental survey in the US. AJGH 2017;100:414-427.

24. Goodman D, Johnson CO, Wenzel L, Bowen D, Condit C, Edwards KL consent issues in genetic research: views of research participants. Public Health Genomics 2016;19:220-228.

25. Nobile H, Bergmann MM, Moldenhauer J, Borry P. Participants' accounts on their decision to join a cohort study with an attached biobank: A qualitative content analysis study within two german studies. J Empir Res Hum Res Ethics 2016;11:237-249.

26. Brothers KB, Morrison DR, Clayton EW. Two large-scale surveys on community attitudes toward an opt-out biobank Am J Med Genet A 2011:155:2982-2990.

27. Schneider UK. Sekundärnutzung klinischer Daten-Rechtliche Rahmenbedingungen: Medizinisch Wissenschaftliche Verlagsgesellschaft: Berlin, Germany, 2015.

28. Harnischmacher U, Ihle P, Berger B, Goebel JW, Scheller J. Checkliste und Leitfaden zur Patienteneinwilligung. Grundlagen und Anleitung für die klinische Forschung, Medizinisch Wissenschaftliche Verlagsgesellschaft: Berlin, Germany, 2006.

29. Prainsack B, Buyx A. Solidarity in biomedicine and beyond. Cambridge University Press: Cambridge, UK, 2017.

30. Edwards T, Cadigan RJ, Evans GP, Henderson GE. Biobanks containing clinical specimens: defining characteristics, Policies, and practices. Clin Biochem 2014;47:245-251

31. Platt J, Bollinger J, Dvoskin R, Kardia SL, Kaufman D. Public preferences regarding informed consent models for participation in population-based genomic research. Genet Med 2014;16:11-18.

32. McCarty CA, Nair A, Austin DM, Giampietro PF. Informed consent and subject motivation to participate in a large, population-based genomics study: the Marshfield Clinic Personalized Medicine Research Project. Community Genet 2007;10:2-9.

33. Ormond KE, Cirino AL, Helenowski IB, Chisholm RL, Wolf WA. Assessing the understanding of biobank participants. Am J Med Genet $A$ 2009;149A:188-198.

34. Rahm AK, Wrenn M, Carroll NM, Feigelson HS. Biobanking for research: a survey of patient population attitudes and understanding. J Community Genet 2013;4:445-450.

35. Paris A, Deygas B, Cornu C, et al. Improved informed consent documents for biomedical research do not increase patients' understanding but reduce enrolment: a study in real settings. Br J Clin Pharmacol 2015;80: 1010-1020.

36. Appelbaum PS, Roth LH, Lidz CW, Benson P, Winslade W. False hopes and best data: consent to research and the therapeutic misconception. Hastings Cent Rep 1987;17:20-24. 